\title{
Phaeocryptopus gaeumannii, patógeno causante del "swiss needle cast" en Pseudotsuga menziesii: antecedentes de su biología, medidas de control y situación en Chile
}

\author{
Phaeocryptopus gaeumannii, pathogen causing the "swiss needle cast” in Pseudotsuga menziesii: \\ biology background, control measures and situation in Chile
}

\author{
Rodrigo Morales a*, Eugenio Sanfuentes ${ }^{\text {b }}$, Isabel Vives a, Eduardo Molina ${ }^{a}$ \\ *Autor de correspondencia: a Universidad Austral de Chile, Facultad de Ciencias Forestales y Recursos \\ Naturales, Instituto de Silvicultura, Valdivia, Chile, tel.: +56-63-221740, rmorales@uach.cl \\ b Universidad de Concepción, Centro de Biotecnología y Facultad de Ciencias Forestales, Concepción, Chile.
}

\begin{abstract}
SUMMARY
Swiss needle cast is a disease caused by Phaeocryptopus gaeumannii, a fungal pathogen causing severe defoliation in Pseudotsuga menziesii (Douglas fir) in some countries of the world, leading to losses in the volume of timber harvesting. This pathogen is distributed in the native range of P. menziesii in North America (U.S., Canada and Mexico) and has been introduced in Europe, New Zealand, Turkey and, recently, in Chile. Currently the country has an area larger than 16,000 ha of plantations of P. menziesii, which are increasing because it is a potentially productive species considered in species diversification programs. In Chile P. gaeumannii is distributed from the region of La Araucanía to Los Lagos, focusing on these areas the largest area of Douglas fir in the country ( $70 \%$ approximately). The aim of this review was to examine studies related to biology and life cycle of the pathogen, epidemiology and infection processes, impact on plantations and control measures implemented in the different countries affected by this pathogen, in order to have a background take on the first studies to Chile, a referent to basic and applied research for the country.
\end{abstract}

Key words: Phaeocryptopus gaeumannii, Pseudotsuga menziesii, volumetric losses timber distribution the SNC in Chile.

\section{RESUMEN}

El swiss needle cast o pérdida suiza de las acículas, es una enfermedad de carácter fungoso provocada por el patógeno Phaeocryptopus gaeumannii, organismo que causa severas defoliaciones en Pseudotsuga menziesii (pino oregón) en el mundo, originando pérdidas en el volumen de madera para cosechar. Este patógeno se distribuye en el rango nativo de P. menziesii en América del Norte (EE.UU., Canadá y México) y se ha introducido en Europa, Nueva Zelanda, Turquía y, recientemente en Chile. Actualmente, el país cuenta con una superficie superior a las 16.000 hectáreas de plantaciones de $P$. menziesii, las cuales van en aumento por tratarse de una especie con potencial productivo considerada en programas de diversificación de especies. En Chile P. gaeumannii se encuentra distribuido desde las regiones de La Araucanía a Los Lagos, concentrándose en estas zonas la mayor superficie de $P$. menziesii del país (70 \% aproximadamente). El objetivo de esta revisión fue analizar estudios relacionados a la biología y ciclo de vida del patógeno, la epidemiología y procesos de infección, impacto en las plantaciones y medidas de control implementadas en los diferentes países afectados por este patógeno, de manera de contar con antecedentes científicos para realizar los primeros estudios en Chile, referentes a investigación básica y aplicada para el país.

Palabras clave: Phaeocryptopus gaeumannii, Pseudotsuga menziesii, pérdidas volumétricas de madera, distribución de SNC en Chile.

\section{INTRODUCCIÓN}

Phaeocryptopus gaeumannii (Rhode) Petrak (sin. Adelopus gaeumannii Rhode) es un patógeno fungoso que causa la enfermedad llamada "swiss needle cast, y/o pérdida suiza de las acículas” (Boyce 1948, Peace 1962, Hansen y Lewis 2003), afectando a Pseudotsuga menziesii (Mirb.) Franco (pino oregón) y a la variedad Pseudotsuga menziesii var. glauca (Mirb.) Franco (Beissn.) Franco, causándoles severas defoliaciones anuales (Hernández 2009, Watt et al. 2010). Esta enfermedad fue reportada por primera vez en Suiza en 1925, afectando plantaciones de P. men- ziesii denominada en alemán schweiser douglasienschütte (defoliación suiza del pino oregón). Luego en 1928, fue observada en Inglaterra y en 1931 en Alemania, posterior a ello se le asigna el nombre en inglés swiss needle cast (Peace 1962). En Alemania y Francia se le asignó a esta enfermedad nombres relacionados con el aspecto negruzco, como hollín, observado sobre el envés de las acículas, denominándola russige douglasienschütte (en alemán, defoliación carbonosa del pino oregón) y rouille suisse $d u$ Douglas (en francés, roya suiza del pino oregón) (Osorio 2007). Gäumann (1951) señala que esta enfermedad era conocida en Irlanda, Inglaterra y Suiza, y que en 1927 se 
expande como pandemia en Europa, motivo que hizo dudar el cultivo del P. menziesii en esos territorios. Este patógeno se encuentra en todo el rango natural de $P$. menziesii en Estados Unidos, Canadá y México. En EE.UU. y Canadá fue reportado en 1938, en Nueva Zelanda en 1959 en P. menziesii var menziesii en el centro de la Isla del Norte, en Turquía el 2006, y en Chile detectado el año 2007 en plantaciones de P. menziesii en Valdivia (Hood y Kershaw 1975, Hood et al. 1990, Hansen et al. 2000, Temel et al. 2003, Osorio 2007).

Pseudotsuga menziesii es un árbol de importancia económica forestal, por la calidad de su madera y usos múltiples en la construcción y mueblería, por ser una especie de rápido crecimiento y resistente a climas fríos (INFORCONAF 1998, Quiroz y Rojas 2003). Estas cualidades han llevado a esta conífera a ser plantada en varios países del mundo, donde P. gaeumannii ha causado perjuicios para su cultivo, reduciendo la superficie foliar, y por ende disminuyendo la productividad de los bosques (Hansen et al. 2000, Manter et al. 2000, Kimberley et al. 2011). Por este motivo, países como EE.UU. y Nueva Zelanda han trabajado por décadas en investigaciones logrando resultados relacionados al estudio de la biología, mecanismos de infección, epidemiología, pérdidas ocasionadas en las plantaciones y su control. En este último punto, los métodos de control empleados han apuntado al control químico, selección genética de árboles resistentes, manejo silvícola y modelos predictivos asociados a variables climáticas y de sitio (McDermott y Robinson 1989, Stone y Coop 2006, Stone et al. 2007).

En EE.UU. en 1997 se crea una cooperativa para abordar investigaciones y extensión de la enfermedad hacia las empresas llamada Swiss Needle Cast Cooperative (SNCC), iniciativa desarrollada por la Universidad del Estado de Oregón en conjunto con empresas forestales y entidades gubernamentales. Actualmente esta cooperativa es una de las entidades que mayor investigación ha desarrollado respecto a la enfermedad (SNCC 2011).

En Chile, son pocos los estudios realizados ligados a P. gaeumannii, determinando la distribución en el país, manejo en laboratorio, y estudios relacionados a la epidemiología del patógeno (Morales 2012).

Los objetivos de esta revisión son analizar investigaciones realizadas en otros países respecto de $P$. gaeumannii sobre el ciclo de vida del patógeno, enfocado a epidemiología y procesos de infección, dimensionar los impactos que este hongo ocasiona en plantaciones de P. menziesii, medidas de control desarrolladas en los países afectados por P. gaeumannii, y proponer acciones a realizar en Chile.

\section{TAXONOMÍA DEL PATÓGENO}

Phaeocryptopus gaeumannii es un hongo perteneciente a la División Ascomycota, orden Dothideales, familia Dothideomycetidae (Winton et al. 2007, Hernández 2009). Estudios moleculares lo han clasificado en la familia Mycosphaerellaceae orden Capnodiales, con las especies Mycosphaerella y Rasutoria (Witnon et al. 2007). Actualmente esta clasificación está siendo mayormente aceptada por la comunidad micológica y patológica de plantas, incluso en aislamientos se observa similitud de $P$. gaeumannii en cultivos in vitro ${ }^{1}$ con patógenos del género Mycosphaerella.

Este hongo se caracteriza por presentar fructificaciones denominadas pseudotecios, que son estructuras de color negro, globosas y lisas, que emergen desde el interior de los estomas, y sus dimensiones varían entre 50 a $80 \mu \mathrm{m}$ de diámetro (Funck 1985, Stone et al. 2008). Los ascos son bitunicados de forma clavada a ligeramente ventricosos, que varían entre 30-40 $\mu \mathrm{m}$ de largo y 8-15 $\mu \mathrm{m}$ de ancho (Funck 1985), y con un máximo de 15 ascos en cada pseudotecio (Morton y Patton 1970). Los ascos presentan ocho ascosporas bicelulares, hialinas, algo fusiformes, levemente acinturadas en la porción central, donde se ubica el tabique que separa sus dos células (Osorio 2007, Stone et al. 2008).

Para los aislamientos del patógeno se utilizan medios de cultivos convencionales, siendo el más empleado el agar extracto de malta entre 2 y $3 \%$ (Hood 1977, Capitano 1999). Los aislamientos de P. gaeumannii se pueden realizar sembrando pequeños trozos de acículas desinfestados superficialmente con alcohol y cloro ${ }^{2}$, o utilizando protocolos para el aislamiento de patógenos del complejo Rhytismatales, induciendo la esporulación de las fructificaciones de tal manera de expulsar la descarga de ascosporas en el medio de cultivo. En cultivo P. gaeumannii solo produce micelio compacto de lento desarrollo, de colores grisáceos claros y oscuros (Cibrián-Tobar et al. 2009). A la fecha, no se ha encontrado la fase asexual del patógeno y se asume que la liberación de las ascosporas sería su mecanismo de infección (Stone y Carroll 1985, Stone et al. 2008). En laboratorio se ha determinado que su desarrollo es óptimo a $20^{\circ} \mathrm{C}$ y decae drásticamente a $30^{\circ} \mathrm{C}$ (Michaels y Chastagner 1984).

Para los ensayos de patogenicidad se han empleado suspensiones de micelio asperjando el follaje de plantas de vivero de $P$. menziesii en los brotes primaverales logrando resultados de infección de un 94 \% posterior a 20 meses (Hood 1977).

Los mecanismos de infección de P. gaeumannii comienzan con la llegada de las ascosporas sobre las acículas, germinando y ramificándose sobre la superficie de éstas, formándose en cada extremo de esta ramificación hifal, estructuras denominadas apresorios sobre cada cavidad estomática que alcanzan. De cada apresorio se origina una hifa infectiva que se introduce en el interior del estoma, comenzando el proceso de infección. El desarrollo interno y colonización de tejidos por parte de $P$. gaeumannii es intercelular a través del mesófilo y, es posible observar hifas del patógeno a los dos meses de ocurrido el proceso

\footnotetext{
Jeff Stone. Profesor Oregon State University. Comunicación personal 2009.

2 Idem.
} 
de infección, siendo más notorias entre los cuatro y nueve meses (Capitano 1999, Stone et al. 2008).

La formación de pseudotecios comienza durante los primeros meses de otoño en brotes infectados en la temporada primaveral pasada (Ford y Morton 1971, Hood 1982, Stone y Carroll 1985, Stone et al. 2008). Se inician con la formación de primordios pseudoteciales bajo las cavidades estomáticas, mediante la diferenciación de las hifas intercelulares. Grupos de cuatro a seis células se alinean bajo y a lo largo de la cavidad estomática, paralelas a las células oclusivas de los estomas, probablemente manteniendo presión en el interior del estoma. Stone et al. 2008 señalan que en el extremo de estas células se presentan engrosamientos periclinales similares a la formación de fiálides, que probablemente corresponden a un estado anamorfo de $P$. gaeumannii, aunque no se observó presencia de conidios. A medida que los pseudotecios aumentan de tamaño, sobre éstos, van quedando pequeños trozos de ceras protectoras de los estomas, los cuales son fáciles de observar en las acículas bajo lupa (Stone y Carroll 1985). Un aspecto interesante y nuevo en la epidemiología del patógeno es el citado por Stone et al. 2008, donde describen la emergencia de hifas de hábito epífito que se desarrollan sobre la superficie de las acículas. Estas hifas son de paredes gruesas, septadas y de pigmentaciones oscuras, que comienzan a aparecer a inicios de verano y continúan proliferando en invierno. Se presume que su función es producir reinfecciones en estomas que no presentan infección, incluso se ha determinado que estas hifas pueden generar redes hifales sobre la superficie de las acículas con la capacidad de producir nuevos apresorios. Sin embrago, aún quedan interrogantes por resolver referente a estos nuevos aspectos relacionados a los mecanismos de infección. Sería interesante verificar si realmente estas hifas epífitas cumplen un rol efectivo en la infección o reinfección en las acículas (Stone et al. 2008).

\section{EPIDEMIOLOGÍA Y DAÑOS EN PLANTACIONES}

El efecto principal del swiss needle cast es la inducción de la abscisión o caída prematura de las acículas producto de la actividad de nutrición del patógeno, causado por una clorosis progresiva y posterior defoliación de los árboles (Hood 1982, Sinclair et al. 1987, Hansen et al. 2000, Kimberley et al. 2011). La vida de las acículas de $P$. menziesii es de siete años, y con ataques sucesivos pueden permanecer menos de cinco años en el árbol, y en ataques más severos sólo pueden permanecer las acículas del último período vegetativo (Hansen y Lewis 2003). Esta defoliación es ocasionada por la oclusión de los estomas dificultando el intercambio gaseoso normal y la absorción de $\mathrm{CO}_{2}$ (Manter et al. 2000, Manter 2002). Se ha estimado que la oclusión del $20 \%$ de los estomas puede reducir aproximadamente en 20 \% la conducción estomática normal, y la asimilación neta de $\mathrm{CO}_{2}$ se reduce diariamente en $60 \%$ (Manter et al. 2000, 2003). Los resultados demues- tran que la defoliación es directamente proporcional al porcentaje de estomas ocluidos por pseudotecios (Hansen et al. 2000). Resulta complejo determinar el grado de severidad del ataque en rodales de $P$. menziesii, por lo que se ha optado por generar índices porcentuales de oclusión estomática, cuantificando la relación entre estomas ocluidos con estomas sin ocluir (Hansen et al. 2000). Otros métodos de evaluación desarrollados tienen relación con técnicas moleculares y cuantificación de ergosterol del patógeno en las acículas infectadas (Winton et al. 2007). Ambos métodos son efectivos y muestran relaciones directamente proporcionales entre los valores obtenidos versus el porcentaje de pseudotecios en las acículas (Manter et al. 2003).

El período de infección de $P$. gaeumannii en Nueva Zelanda se inicia en primavera con la maduración de pseudotecios, y con un máximo de esporulación en octubre y noviembre coincidente con el inicio de elongación de brotes de $P$. menziesii, los primeros pseudotecios aparecen a comienzos de otoño. En EE.UU., en el Estado de Michigan, se determinó una tendencia similar en cuanto a la esporulación e infección estacional ocurrida en Nueva Zelanda (Ford y Morton 1971, Hood 2004). Solo se observa una leve diferencia en estos procesos existiendo un desfase de un mes entre ambos hemisferios, ocurriendo un mes antes el máximo de esporulación en Nueva Zelanda a diferencia de EE.UU. Esta diferencia podría estar explicada por las condiciones climáticas de cada lugar geográfico, especialmente el comportamiento de las temperaturas de invierno y llegada de la primavera. En EE.UU. en zonas donde se desarrolla naturalmente $P$. menziesii, las temperaturas de inviernos pueden alcanzar $-18^{\circ} \mathrm{C}$, y el aumento de las temperaturas hacia la primavera es lenta y gradual. Esta condición podría estar afectando la maduración de los pseudotecios y podría explicar la diferencia de liberación anticipada de ascosporas en Nueva Zelanda, que presenta un clima templado con influencia marina (Ford y Morton 1971, Stone et al. 2007).

Los niveles de infección de $P$. gaeumannii son muy fluctuantes de una región a otra y están sujetos a variables relacionadas con el clima, principalmente, la precipitación y temperatura (Hood 1982, Manter et al. 2005, Stone et al. 2007). El agua caída e infección de los árboles presenta fuerte correlación con precipitaciones mensuales entre 250-350 mm, produciéndose fuertes niveles de infección en los árboles, entre 80 y 100 \% (Hood 1982). Se ha determinado que la influencia de las temperaturas mínimas de invierno presentan mayor correlación con la infección y progresión de la enfermedad ocurrida en la primavera del año en curso (Stone et al. 2007). También se señala que la altitud incide en el desarrollo y niveles de severidad del patógeno, concluyendo que altitudes bajo los $200 \mathrm{~m}$ s.n.m. los rodales presentan mayor infección (Kaster et al. 2001, Manter et al. 2005.

En la década de los noventa, los daños en árboles de navidad se intensificaron drásticamente a lo largo de la costa 
de Oregon y Washington (Filip et al. 2000). Los mayores daños se han reportado en árboles de navidad en EE.UU. en el Estado de Washington, con pérdidas de 3,4 millones de dólares (600.000 árboles) presentando diferentes niveles de daño (Michaels y Chastagner 1984). En 1999 a través de prospecciones aéreas se determinó que de un total de 1,2 millones de hectáreas evaluadas en la costa del Estado de Oregón, 119.387 hectáreas (alrededor del 10 \% de la superficie) fueron categorizadas con infección severa (Hansen et al. 2000). Similar evaluación efectuada en la costa de Washington estimó 80.940 hectáreas infectadas severamente de 930.810 hectáreas (Filip et al. 2000).

En Nueva Zelanda, desde su primer reporte en 1959, $P$. gaeumannii se ha expandido por toda la isla, y se han realizado evaluaciones en parcelas permanentes durante 30 años, para estimar pérdidas económicas asociadas al patógeno (Kimberley et al. 2011). Los resultados obtenidos muestran una disminución constante en la tasa de crecimiento de los rodales evaluados. La reducción del crecimiento promedio acumulado de los árboles para la altura total fue de 25 \%, para el área basal 27 \% y el volumen total de la troza fue de un $32 \%$ de reducción. Las pérdidas económicas se estiman aproximadamente en US\$ 3.000/ $\mathrm{ha}^{-1}$ para la isla del norte y US\$1.700/ ha-1 para la isla del sur (Kimberley et al. 2011).

\section{MEDIDAS DE CONTROL}

Las medidas de control desarrolladas han comprendido el control químico, selección genética de árboles resistentes, manejo silvicultural y modelos predictivos asociados a variables climáticas y de sitio (Hood y Van der Pas 1979, Filip et al 2000, Johnson 2002, Mainwaring 2005, Manter et al. 2005, Temel et al. 2005, Watt et al. 2010).

Control químico. Se ha utilizado el óxido cuproso como fungicida en plantas de vivero de $P$. menziesii, en intervalos aproximados de 25 días durante el crecimiento de los brotes en primavera, con eficacia entre 60 y $70 \%$ aproximadamente (Hood 1976). En árboles de navidad se ha utilizado clorotalonil, demostrando eficacia en aplicaciones primaverales durante el desarrollo de los árboles (Chastagner y Byther 1983). En plantaciones forestales jóvenes y adultas de $P$. menziesii, se han utilizado productos con ingredientes activos como el clorotalonil, carbendazima y cobre, aplicando de forma aérea y reduciendo la infección entre un 55 y 93 \% (Hood y Van der Pas 1979, Stone et al. 2007). Estos resultados demuestran un control eficaz de $P$. gaeumannii; sin embargo, la aplicación prolongada de químicos en el tiempo y lo extenso de las superficies resulta económicamente inviable, y ambientalmente podrían traer consecuencias nocivas para el ecosistema natural (Hansen y Lewis 2003, Stone et al. 2007).

Selección genética. En bosques de P. menziesii la resistencia al ataque por $P$. gaeumannii se puede observar en diferentes procedencias y dentro de una misma procedencia. Estas características han sido determinadas en función de variables dasométricas (DAP, área basal, altura, volumen) y características del follaje de los árboles (retención de las acículas, color, oclusión de estomas, entre otras) mostrando antecedentes de resistencia al patógeno, siendo una alternativa factible de control al mediano y largo plazo (McDermontt y Robinson 1989, Johnson 2002, Temel 2002, Temel et al. 2005). El material genético con mayores atributos de resistencia está localizado en la zona costera entre la Columbia Británica y Washington. En esta región los bosques de $P$. menziesii se encuentran en su rango natural de distribución coexistiendo con otras especies, a diferencia de las procedencias de California que presentan mayor susceptibilidad, existiendo reportes superiores al 90 \% de infección (McDermontt y Robinson 1989, Hood et al. 1990). Nelson et al. (1989) ratificaron también alta susceptibilidad al patógeno en familias de P. menziesii procedentes de la costa de California pero, probablemente las condiciones de precipitación y humedad relativa de California también contribuyen en fomentar la infección del patógeno (Hansen y Lewis 2003).

Control silvicultural. En este control se han probado métodos de regeneración, raleos, podas, control de malezas y fertilizaciones (Hood y Sandberg 1979, Filip et al. 2000, El-Hajj et al. 2004, Mainwaring et al. 2005).

A través de los métodos de regeneración en bosques naturales de árbol semillero y regeneración natural y cortas selectivas, se ha verificado que en las zonas donde $P$. menziesii crece de manera natural y mixto con otras especies (Picea sitchensis (Bong.) Carrière, Tsuga heterophylla (Raf.) Sarg.) los niveles de daños y la presencia de $P$. gaeumannii son menos severos, a diferencia de rodales puros en otras zonas geográficas (Filip et al. 2000).

El control de malezas se ha planteado a modo de hipótesis posibles prácticas que pueden reducir el daño por $P$. gaeumannii en la regeneración natural o en plantaciones recién establecidas y jóvenes. Se piensa que las plantas sin competencia por malezas frente a eventuales ataque por $P$. gaeumanii, el impacto del crecimiento y desarrollo por las defoliaciones en los árboles debería ser menor, por contar con mayor disponibilidad de agua, luz, y nutrientes. Aunque esta hipótesis no está comprobada, se recomienda el control de malezas para garantizar mayor vigor en las plantas frente a ataques de cualquier tipo de organismos (Filip et al. 2000).

Referente a la fertilización en plantaciones de $P$. menziesii, los fertilizantes nitrogenados como la urea después de raleos precomerciales, han generado incrementos en el desarrollo de fructificaciones y daño por $P$. gaeumannii (Filip et al. 2000, El-Hajj et al. 2004). Por este motivo se recomienda una fertilización balanceada con otros elementos, determinados a través de análisis de suelo y foliar. Actualmente se están probando otros elementos, como calcio, azufre y magnesio, para estudiar su efecto en la severidad de $P$. gaeumannii (Filip et al. 2000). 
En plantaciones comerciales se ha observado que rodales afectados por P. gaeumannii responden positivamente en incremento volumétrico por árbol individual, independiente del nivel de infección que tengan los rodales. Esta medida silvícola puede ser usada para recuperar aquellos rodales que presentan severos niveles de infección, con el fin de mejorar la productividad de los rodales (Mainwaring et al. 2005).

Modelos predictivos. Esta herramienta se basa en la predicción de ocurrencia y severidad del daño que pudiera causar el patógeno en diferentes zonas geográficas en un horizonte de tiempo (Rosso y Hansen 2003, Manter et al. 2005). En el oeste de Oregón (EE.UU.) las temperaturas mínimas de invierno, precipitaciones medias mensuales y mojamiento foliar, explican alrededor de $80 \%$ la severidad P. gaeumanii (Stone y Coop 2006). En EE.UU. y Nueva Zelanda han utilizado esta herramienta, simulando escenarios posibles de clima referentes al cambio climático global (Stone y Coop 2006, Watt et al. 2010). En ambos países se ha concluido que las variaciones del clima en cuanto a un mayor aumento de las precipitaciones y temperaturas podrían incrementar un comportamientos más agresivo de $P$. gaeumanii que los observados en años normales, pudiendo ocasionar potenciales impactos en la productividad de los bosques de P. menziesii (Hood 1982, Stone y Coop 2006, Watt et al. 2010).

\section{SITUACIÓN EN CHILE}

Phaeocryptopus gaeumannii fue reportado por primera vez en Chile el año 2007 en la región de Los Ríos (Osorio 2007). Se detectó en plantaciones de P. menziesii de diferentes edades, inclusive en viveros en plantas 2/0. Esta ocurrencia de $P$. gaeumannii en un amplio rango de edades de las plantaciones genera un riesgo en la productividad de $P$. menziesii, considerando que es una de las especies emergentes para los programas de diversificación forestal del país, y podrían generarse pérdidas volumétricas y económicas considerables (INFOR-CONAF 1998, Kimberley et al. 2011).

La distribución de $P$. gaeumannii en Chile, actualmente comprende desde las regiones de La Araucanía a Los Lagos (Morales 2012) (figura 1). Estas regiones concentran la mayor superficie de $P$. menziesii del país, con un $70 \%$ del total, aproximadamente (INFOR-CONAF 1998), lo que implica un riesgo en la productividad de estos bosques.

En laboratorio se han realizado los primeros aislamientos del patógeno, encontrando diferencias morfológicas de cepas en cuanto a su color (figura 2) (Morales 2012).

En los aislamientos se encontró la presencia de dos tipos de colonias (figura $2 \mathrm{~B}$ ), una de aspecto blanquecino al inicio y durante su crecimiento que se definió como tipo A y otra de color gris oscuro a negro definida como tipo B (Morales 2012). La particularidad de estas colonias, es que en un mismo lugar de colecta se pueden aislar ambos

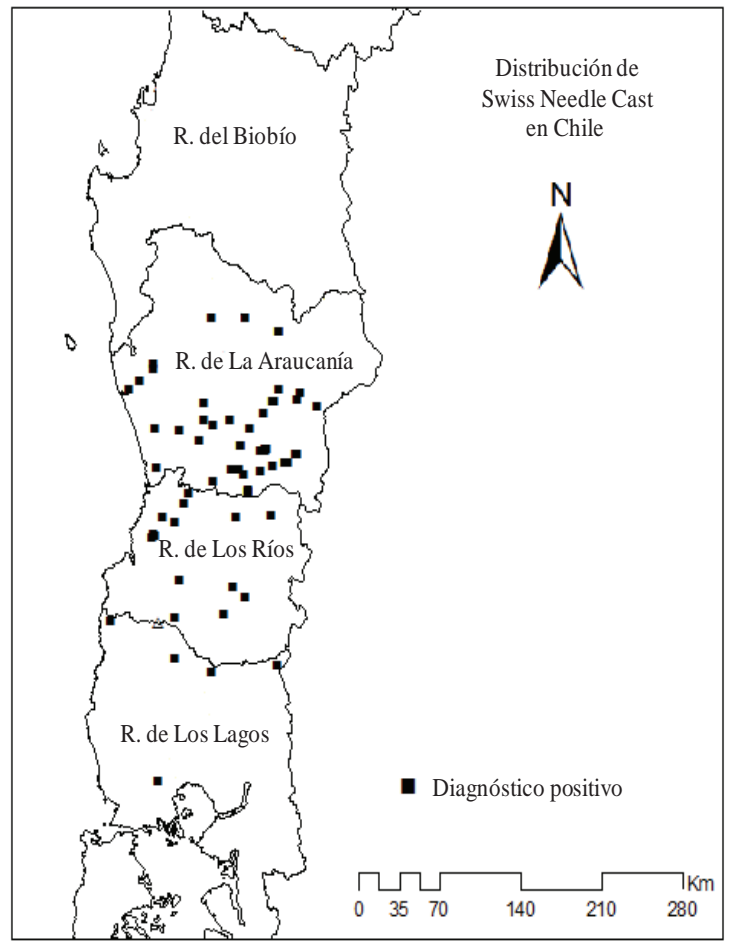

Figura 1. Distribución del swiss needle cast (Phaeocryptopus gaeumannii) en Chile (Morales 2012). mannii) in Chile.

Distribution of Swiss needle cast (Phaeocryptopus gaeu-

tipos. Esta característica lleva a pensar que podría existir diversidad poblacional de cepas ligada a características patogénicas del hongo, por lo cual habría que corroborarlo a través de análisis moleculares y pruebas de patogenicidad. Si bien no hay literatura relacionada a estas diferencias morfológicas, en EE.UU y Nueva Zelandia se ha observado similar particularidad en los aislamientos ${ }^{3}$, pero no se han realizado estudios al respecto, que aporten a dilucidar estas diferencias.

Los estudios básicos a realizar en el corto plazo, deberían enfocarse al estudio del ciclo de vida del patógeno, epidemiología y variabilidad poblacional, para establecer futuras medidas de manejo de la enfermedad, el control químico en vivero, debería ser la medida más pronta a aplicar en viveros, ya que Osorio (2007) detectó P. gaeumannii en plantas $2 / 0$. Teniendo en consideración la distribución actual del patógeno en Chile, probablemente se podría dispersar su distribución geográfica por motivos de comercialización de plantas hacia otras regiones del país sin presencia de $P$. gaeumannii.

Para las plantaciones de $P$. menziesii el manejo silvícola y el promover cultivos mixtos de esta especie, según

\footnotetext{
3 Jeff Stone. Profesor de la Oregon State University. Comunicación personal 2010.
} 

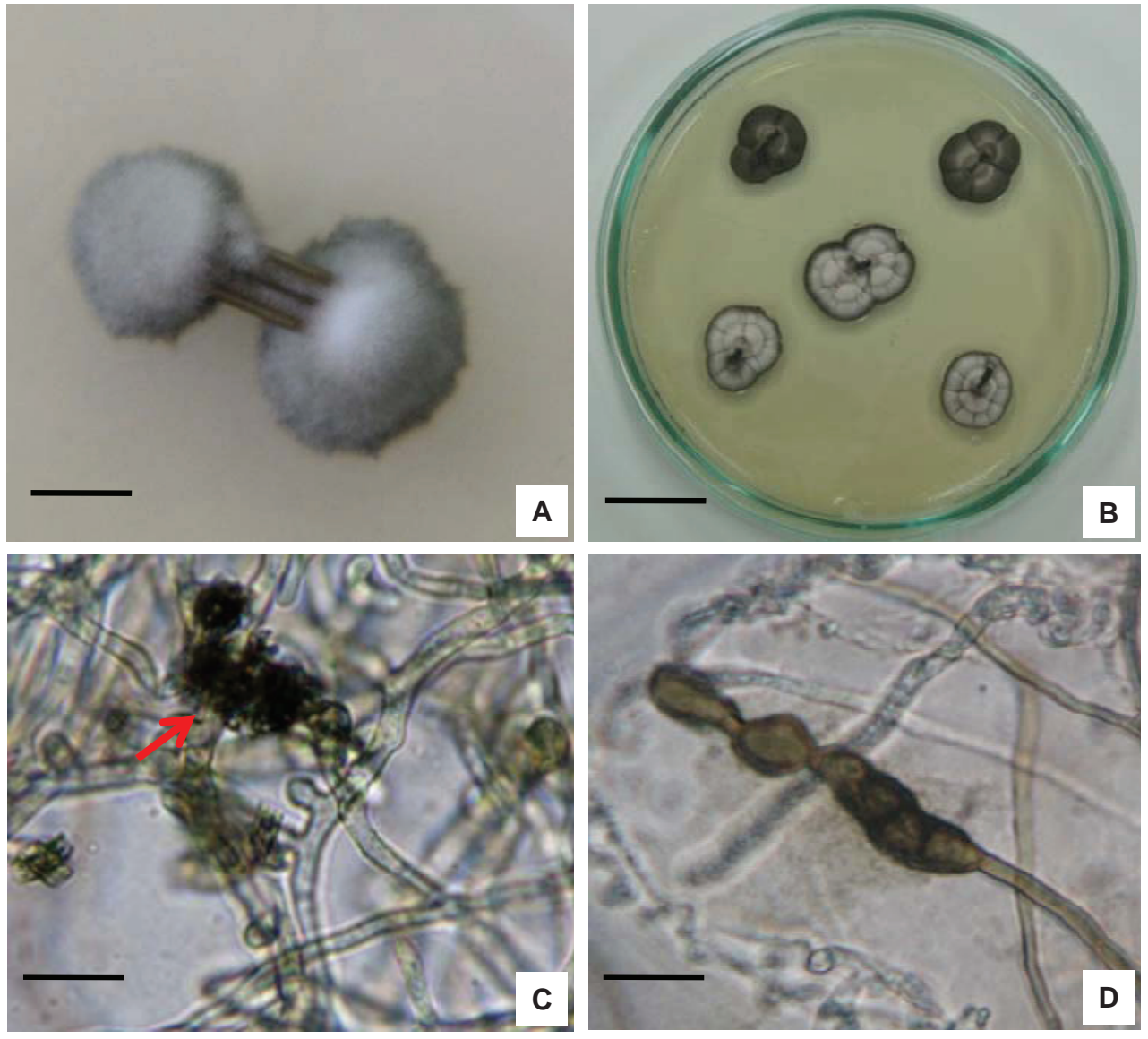

Figura 2. Aislamientos y características de las colonias de $P$. gaeumannii. A) Aislamiento a partir de un trozo de acícula infectada (barra representa $1 \mathrm{~mm}$ ); B) Diferencias morfológicas de color de las cepas de P. gaeumannii (barra representa $10 \mathrm{~mm}$ ) C) Desarrollo de micelio y aglomeraciones hifales (flecha roja) a los 20 días en cultivo de agar malta $2 \%$ (barra representa $10 \mu \mathrm{m}$ ); D) Formación de clamidósporas en el extremo de hifas después de 40 días en agar malta 2 \% (barra representa $10 \mu$ m) (Morales 2012).

Characteristic and isolates of strains P. gaeumannii. A) Isolate of infected needle portion (the bar represents $1 \mathrm{~mm}$ ); B) Morphological difference of strains colors (bar represents $10 \mathrm{~mm}$ ); C) Growth mycelium and agglomeration (red arrow) in malt extract agar $2 \%$ in 20 days (bar represents $10 \mu \mathrm{m}$ ); D) Chlamydospores formation at the tip of the hypha in 40 days after in malt extract agar $2 \%$ (bar represents $10 \mu \mathrm{m}$ ).

la experiencia norteamericana, disminuye los índices de infección, junto a intervenciones a los raleos oportunos, para fomentar vigorosidad y ventilación del rodal para evitar condiciones favorables para el desarrollo del patógeno.

En el mediano y largo plazo la selección genética de individuos resistentes parece representar las líneas de investigación más apropiadas para el país en el futuro para enfrentar la enfermedad, de manera de minimizar las defoliaciones y pérdidas de volumen de madera.

\section{AGRADECIMIENTOS}

Los autores agradecen a la Dirección de Investigación de la Universidad de Concepción (DIUC) por el financiamiento de este trabajo en el marco del proyecto DIUC 209.005.002-1.0 "Estudios del patógeno Phaeocryptopus gaeumannii asociado a defoliaciones de Pseudotsuga menziesii en Chile”. Al Dr. Jeff Stone profesor de Oregon
State University, EE.UU., y al Dr. Ian Hood investigador de SCION, Nueva Zelanda, por sus apoyos bibliográficos para esta revisión.

\section{REFERENCIAS}

Boyce J. 1948. Forest Pathology. $2^{\mathrm{a}}$ ed. New York, USA. McGraw-Hill. 550 p.

Capitano B. 1999. The infection and colonization of Douglas-fir by Phaeocryptopus gaeumannii. Tesis Magíster en Ciencias. Corvallis, Oregon, Estados Unidos. Departamento de Botánica y Patología de Plantas, Universidad Estatal de Oregón. $81 \mathrm{p}$.

Chastagner G, R Byther. 1983. Control of Swiss needle cast on Douglas fir Christmas trees with aerial applications of chlorothalonil. Plant Disease 67: 790-792.

Cibrián-Tobar D, O Pérez-Vera, S García-Díaz, J Cruz-Juárez. 2009. Caracterización morfológica y molecular del tizón suizo (Phaeocryptopus gaeumannii) en Pseudotsuga men- 
ziesii var glauca en México. In Memoria del XV Simposio Nacional de Parasitología Forestal. Oaxaca, México,18 al 20 de noviembre. 265 p.

El-Hajj Z, K Kavanagh, C Rose, Kanaan-Atallah. 2004. Nitrogen and carbon dynamics of a foliar biotrofic fungal parasite in fertilized Douglas-fir. New Phytologist 163: 139-147.

Filip G, A Kanaskie, K Kavanagh, G Johnson, R Johnson, D Maguire. 2000. Silviculture and Swiss needle cast: Research and recommendation. Oregon, EE.UU. College of Forestry, Oregon State University. 16p.

Ford K, H Morton. 1971. Etiology, impact, and distribution of Swiss needle cast of Douglas-fir in Michigan. In abstract of the 1971 Annual Meeting of the North Central Division of American Phytopathological Society the 13-14 July in East Lansing, Michigan. Phytopathology 61:1022-1026.

Funk A. 1985. Foliar fungi of western trees. Canadá. Canadian Forestry Service, Pacific Forest Research Centre. 159 p.

Gäumann E. 1951. Pflanzliche Infektionslehre: Lehrbuch der allgemeinen Pflanzpathologie für Biologen, Landwirte, Förste und Pflanzenzüchter. Birhäusr, Basel, Suiza. 681 p.

Hansen E, K Lewis. 2003. Plagas y enfermedades de coníferas. The American Phytopathological Society. Madrid, España. Ediciones Mundi-Prensa. 101 p.

Hansen E, J Stone, B Capitano, P Rosso, W Sutton, L Winton, A Kanaskie, M McWilliams. 2000. Incidence and Impact of Swiss Needle Cast in Forest Plantations of Douglas-fir in Coastal Oregon. Plant Disease 84: 773-778.

Hernández G. 2009. Determinación del periodo de liberación de ascoporas de Phaeocryptopus gaeumannii (Rhode) Petrak en plantaciones de árboles de navidad. Tesis de Ingeniero Forestal. Chapingo, México. División de Ciencias Forestales, Universidad Autónoma de Chapingo. 75p.

Hood I. 2004. Swiss Needle Cast Disease. The New Zealand Experience. Presentation. Conference: Growing Douglas- Fir in the Swiss Needle Cast Zone, Oregon State University. Oregon. 16 de noviembre, 2004. Consultado 02 feb. 2011. Disponible en http://outreach.forestryoregonstate.edu/snc/ agenda.htm.

Hood I, C Samberg, C Barr, W Holloway, P Bradbury. 1990. Changes in needle retention associated with the spread and establishment of Phaeocryptopus gaeumannii in planted Douglas fir. European Journal Forest Pathology 20: 418429.

Hood I. 1982. Phaeocryptopus gaeumannii on Pseudotsuga menziesii in southern British Columbia. New Zealand Journal of Forestry Science 12: 415-424.

Hood I, J van der Pas. 1979. Fungicidal control of Phaeocryptopus gaeumanni in a 19-year-old Douglas fir stand. New Zealand Journal of Forestry Science 9: 272-83.

Hood I. 1977. Inoculation experiments whit Phaeocryptopus gaeumannii on Douglas-fir seedling. New Zealand Journal of Forest Science 7: 77-82.

Hood I. 1976. Investigations into the fungicidal effect of cuprosus oxide againt Phaeocryptopus gaeumannii on Douglasfir seedling. New Zealand Journal of Forestry 21(1): 141145.

Hood I, D Kershaw. 1975. Distribution and infection period of Phaeocryptopus gaeumannii in New Zealand. New Zealand Journal of Forestry Science 5: 201-208.

INFOR (Instituto Forestal, CL)-CONAF (Corporación Nacional Forestal, CL). 1998. Monografía de pino oregón, Pseudot- suga menziesii. Santiago, Chile. Neuenschwander y Cruz. $143 \mathrm{p}$.

Johnson G. 2002. Genetic variation in tolerance of douglas-fir to Swiss needle cast as assessed by symptom expression. Silvae Genetica 51: 80-86.

Kaster W, S Dutton, D Roché. 2001. Effects of Swiss needle cast on three Douglas-fir seed sources on a low-elevation site in the northern Oregon coast range: Result after five growing seasons. Western Journal of applied Forestry 16(1): 31-34.

Kimberley M, I Hood, R Knowles. 2011. Impact of Swiss needle-cast on growth of Douglas-fir. Phytopathology 101(5): 583-593.

Mainwaring D, D Maguire, A Kanaskie, J Brand. 2005. Growth responses to commercial thinning in Douglas-fir stands whith varying severity of Swiss needle cast in Oregon, USA. Canadian Journal of Forest Research 35: 2394-2402.

Manter D, P Reeser, J Stone. 2005. A climate-based model for predicting geographic variation in Swiss needle cast severety in the Oregon coast range. Phytopathology 95(11): 1256-1265.

Manter D, B Bond, K Kavanagh, J Stone, G Filip. 2003. Modelling the impacts of the foliar pathogen, Phaeocryptopus gaeumannii, on Douglas-fir physiology: net canopy carbon assimilation, needle abscission and growth. Ecological Modeling 164: 211-226.

Manter D. 2002. Energy dissipation and photoinhibition in Douglas-fir needles with a fungal-mediated reduction in photosynthetic rates. Journal Phytopathology 150: 674-679.

Manter D, B Bond, K Kavanagh, P Rosso, G Filip. 2000. Pseudothecia of Swiss needle cast fungus, Phaeocryptopus gaeumannii, physically block stomata of Douglas-fir, reducing $\mathrm{CO}_{2}$ assimilation. New Phytology 148: 481-491.

McDermott J, R Robinson R. 1989. Provenance variation for disease resistance in Pseudotsuga menziesii to the Swiss needle cast pathogen Phaeocryptopus gaeumannii. Canadian Journal Forest Research 19: 244-246.

Michaels E, G Chastagner. 1984. Seasonal availability of Phaeocryptopus gaeumannii ascospores and conditions that influence their release. Plant Disease 68(11): 942-944.

Morales R. 2012. Estudios del patógeno Phaeocryptopus gaeumannii asociado a defoliaciones en plantaciones de Pseudotsuga menziesii en Chile. Tesis Doctorado en Ciencias Forestales. Valdivia, Chile. Facultad de Ciencias Forestales y Recursos Naturales, Universidad Austral de Chile. 85 p.

Morton H, R Patton. 1970. Swiss needle cast of Douglas-fir in the Lake States. Plant Disease Report 54(7): 612-616.

Nelson E, R Silen, N Mandel. 1989. Effect of Douglas-fir parentage on Swiss needle cast expression. European Journal Forest Pathology 19:1-6.

Osorio M. 2007. Detección del hongo defoliador Phaeocryptopus gaeumannii en plantaciones de Pseudotsuga menziesii de Valdivia, Chile. Bosque 28(1): 69-74.

Peace T. 1962. Pathology of trees and shrubs. With special reference to Britain. Clarendon, Oxford, London. University Press, Amen House,.757 p.

Quiroz I, Y Rojas. 2003. Pino ponderosa y Pino oregón: Coníferas para el sur de Chile. Valdivia, Chile. Instituto Forestal, Sede Los Lagos. 316 p.

Rosso P, E Hansen 2003. Predicting Swiss Needle Cast Disease Distribution and Severity in Young Douglas-Fir Plantations in Coastal Oregon. Phytopathology 93: 790-798. 
Sinclair W, H Lyon, W Johson. 1987. Diseases of tres and shrubs. Comstock, London. 574 p.

SNCC. 2011. Swiss Needle Cast Cooperative. College of Forestry, Oregon State University. Consultado 7 jul. 2011. Disponible en http://sncc.forestry.oregonstate.edu/.

Stone J, I Hood, M Watt, J Kerrigan. 2007. Distribution of Swiss needle cast in New Zealand in relation to winter temperature. Australasian Plant Pathology 36: 1-10.

Stone J, B Capitano, J Kerrigan 2008. The histopatology of Phaeocryptopus gaeumannii on Douglas- fir needle. Mycologia 100(3): 431- 444.

Stone J, L Coop. 2006. Predicting effect of climate change on Swiss needle cast disease severity in Pacific Northwest forest. In Jackson M. 2007. Proceeding of the 54 ${ }^{\text {th }}$ Anuual Western International Forest Disease Work Conference; 2006 october 2-6; Smithers, BC. Missoula, MT: USA. Department of Agriculture, Forest Service, Forest Health Protection. $153 \mathrm{p}$.

Stone J, G Carroll. 1985. Observations of the development of ascocárpos in Phaeocryptopus gaeumannii and on the possible existence of an anamorphic state. Sydowia 38: 317-323.

Temel F, G Johnson, W Adams. 2005. Early genetic testing of coastal Douglas-fir for Swiss needle cast tolerance. Canadian Journal of Forest Research 35: 521-529.

Temel F, J Stone, G Jonson. 2003. First report of Swiss needle cast caused by Phaeocryptopus gaeumannii on Douglas-Fir in Turkey. Plant Disease 87: 1536.

Temel F. 2002. Early testing of Douglas-fir (Pseudotsuga menziesii var. menziesii (Mirb.) Franco) for Swiss needle cast tolerance. Thesis Doctor of Philosophy. Oregon, EE.UU. Department of Forest Science, Oregon State University. 177 p.

Watt M, J Stone, I Hood, D Palmer. 2010. Predicting the severity of Swiss needle cast on Douglas-fir under current and future climate in New Zealand. Forest Ecology and Management 260: 2232-2240.

Winton L, J Stone, E Hansen, R Shoemaker. 2007. The systematic position of Phaeocryptopus gaeumannii. Mycologia 99: 240-252.

Recibido: 04.01.12

Aceptado: 08.06.12 